\title{
Effect of food and water deprivation on hippocampal self-stimulation and poststimulation feeding
}

\author{
N. W. MILGRAM \\ University of Toronto, Toronto, Ontario, Canada \\ ALFRED C. SERVER \\ Stanford University Medical School, Stanford, California 94305 \\ and \\ K. A. CAMPBELL \\ University of Toronto, Toronto, Ontario, Canada
}

\begin{abstract}
Barpressing for electrical stimulation of the dorsolateral hippocampus was facilitated by both food and water deprivation. These results were not simply attributable to a nonspecific effect of deprivation on self-stimulation; food deprivation but not water deprivation produced an increase in barpressing for lateral hypothalamic stimulation. Feeding elicited by dorsolateral hippocampal stimulation increased after food deprivation, but was not affected by water deprivation. These findings suggest a dissociation between the reinforcing consequences of hippocampal stimulation and the effects of hippocampal stimulation on feeding. In addition, there seems to be at least some degree of independence between the neural mechanisms underlying hippocampal reward and those underlying hypothalamic reward.
\end{abstract}

Electrical stimulation of some neural loci can both support positive reinforcement and elicit specific behavioral sequences such as eating or drinking (e.g., Margules \& Olds, 1962; Mogenson \& Stevenson, 1967). Such findings may reflect the simultaneous activation of functionally different neural systems (e.g., Ball, 1969; Deutsch \& Deutsch, 1973). Alternatively, elicited behaviors and self-stimulation may both be consequences of activation of natural reinforcement systems (Hoebel, 1971; Trowill, Panksepp, \& Gandelman, 1969). It is difficult to distinguish between these two alternatives on the basis of the relevant research. One problem is that the large majority of pertinent studies have used reinforcing hypothalamic placements, and the behavioral consequences of hypothalamic stimulation are generally not specific (e.g., Valenstein, Cox, \& Kakolewski, 1970), possibly because of the simultaneous activation of functionally diverse overlapping systems (Milgram, Devor, \& Server, 1971).

The present experiments investigated the relation-

This research was supported in part by United States Public Health Service Grant MH08493 and National Science Foundation Grant GB431X to B. G. Hoebel, in part by National Research Council of Canada Grant A7659 to N. W. Milgram, and in part by National Institute of Mental Health Grant MH11550-04 to A. C. Server. Reprint requests should be addressed to N. W. Milgram, Life Sciences Division, Scarborough College, University of Toronto, West Hill, Ontario, Canada. ship between self-stimulation and elicited behaviors using dorsolateral hippocampal placements. Stimulation of this region both supports self-stimulation and causes a facilitation of feeding which occurs following the offset of the stimulation (Milgram, 1969a; Oliver, Firestone, \& Goodman, 1973). Unlike hypothalamic stimulation, the elicited behavioral consequences of hippocampal stimulation seem specific to feeding: drinking and other behaviors are not facilitated by hippocampal stimulation, even when special training procedures are employed (Milgram, 1969b). If hippocampal self-stimulation was specifically related to the rewarding effects of feeding, then both self-stimulation and poststimulation feeding should be under the control of events which contol normal feeding and not drinking.

\section{EXPERIMENT 1 EFFECT OF FOOD AND WATER DEPRIVATION ON HIPPOCAMPAL SELF-STIMULATION}

\footnotetext{
Subjects

General Methods

Subjects were 11 individually housed male Long-Evans rats which were maintained on ad lib food and water, except when otherwise indicated.

Single monopolar platinum-irridium electrodes, 0.01 in. diam and insulated down to approximately $0.5 \mathrm{~mm}$ of the sharpened tips, were implanted in the dorsolateral hippocampus of each rat, using stereotaxic coordinates $3.5 \mathrm{~mm}$ anterior to the ear
} 
bars, $4.0-4.2 \mathrm{~mm}$ lateral to the midline, and $3.3 \mathrm{~mm}$ down from the dura; the electrode path was perpendicular to the surface of the skull.

\section{Testing Apparatus and Training Procedure}

Testing was in a $7 \times 12 \times 12$ in. Plexiglas box fitted with a bar at the narrow end, depression of which delivered a $0.5-\mathrm{sec}$ train of $100-\mathrm{Hz}$ biphasic $0.1-\mathrm{msec}$ square-wave pulses.

The animals were trained to barpress for hippocampal stimulation following a postsurgical recovery period of at least 1 week. The current intensity was individually adjusted to a level which caused hyperactivity following a brief hippocampal stimulation (range 90-400 $\mu \mathrm{A}$ ). Because shaping animals to barpress for hippocampal stimulation is difficult, training was generally accomplished by leaving the animal overnight in the testing box. Up to six such overnight sessions were sometimes necessary.

\section{Tests for Poststimulation Feeding and Histological Verification}

At the conclusion of the experiment, the animals were tested for poststimulation feeding using a self-induced feeding procedure as described by Milgram (1969a). Poststimulation feeding was observed in every animal, and there was no indication of poststimulation drinking.

The electrode loci were verified from coronal sections stained with cresyl violet and were found to terminate in the proximity of the pyramidal cell field CA3 of the dorsal hippocampus.

\section{Experiment 1a Effect of Food Deprivation on Hippocampal Self-Stimulation}

\section{Methods}

Six animals were used in this experiment. After learning to barpress for hippocampal stimulation, the animals were tested for $1 / 2 \mathrm{~h}$ daily. The experimental phase began on the day after barpressing rates had stabilized. Stabilization was assumed if, after three consecutive daily sessions, no single score differed by more than $20 \%$ from the mean score of the three sessions. Twenty-four hours later, the animals were deprived of food for $23 \mathrm{~h}$, and, following a day of ad-lib feeding, food was again removed for a 23 -h period. Thus, food had been available ad lib prior to the barpressing tests on Test Days 1 and 3; a 23-h fooddeprivation period preceded the barpressing test on Test Days 2 and 4.

After the first 4 days of testing, the mean barpressing rate under deprivation was compared with the mean barpressing rate during the two control sessions. If the difference was within the variability observed during the three stabilization sessions, it was concluded that deprivation had no effect at this stimulation intensity and the animals were retested using exactly the same procedure, but at a lower intensity of stimulation. However, if the effect of food deprivation exceeded this criterion, then the testing period was extended to a total of six sessions. The rats had food ad lib prior to the fifth session and were deprived of food prior to the sixth.

\section{Results and Discussion}

Figure 1 shows the daily barpressing rate of a typical animal in order to illustrate the procedure followed and the results typically observed. A clear facilitation of barpressing under food deprivation occurred in all six animals (correlated means, $\mathrm{t}=3.41$, $\mathrm{df}=5, \mathrm{p}<.02$, two-tailed). Five of the six rats showed the increase in barpressing at the current originally selected; it was necessary to lower the

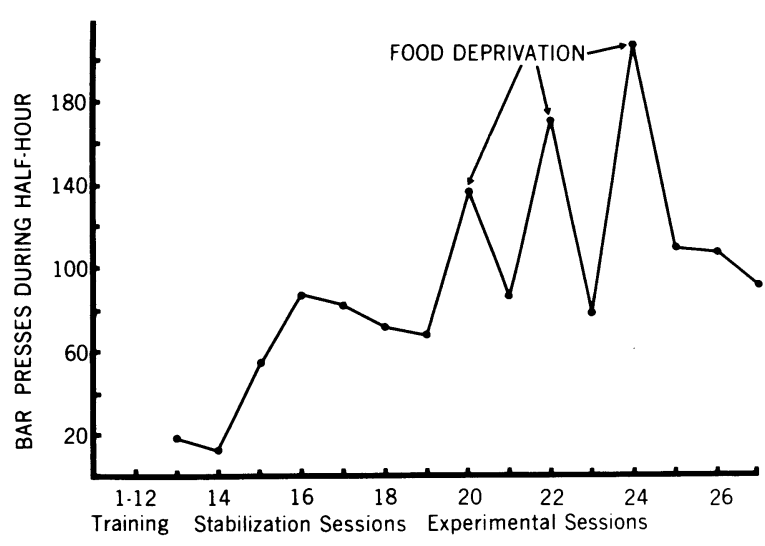

DAY OF TESTING

Figure 1. Daily rates of barpressing for one animal over stabilization and testing sessions.

current once before an increase occurred in the sixth animal.

Figure 2 summarizes the mean barpressing rate during the three stabilization sessions and the six experimental sessions. Even though the control rates did not differ significantly from the stabilization rates, the trend during the experimental sessions of an increase in barpressing is similar to that previously reported for hypothalamic animals tested under similar conditions (Goldstein, Hill, \& Templer, 1970).

\section{Experiment 1b \\ Effect of Water Deprivation on Hippocampal Self-Stimulation}

\section{Methods}

Subjects were nine rats, five of which had been tested in Experiment 1a. The procedure followed was as described for Experiment 1a, except that the animals were deprived of water, not food, on the experimental sessions.

\section{Results and Discussion}

Eight of the nine animals barpressed at higher

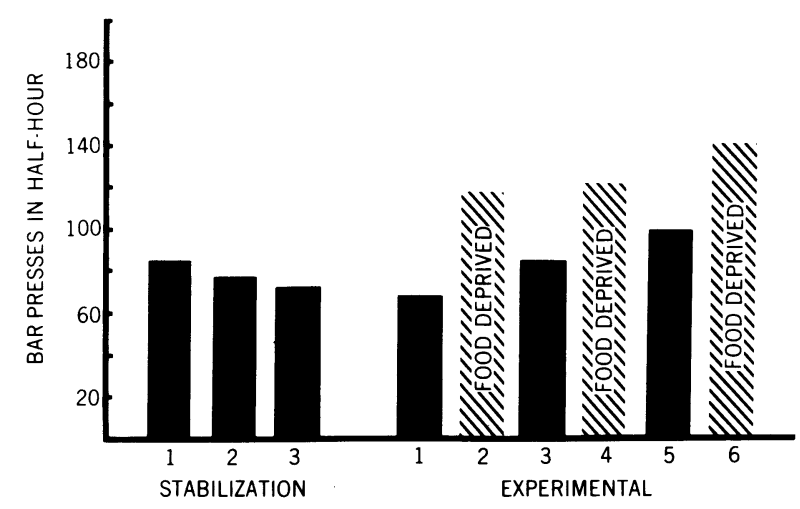

Figure 2. Mean barpresses for hippocampal stimulation in $1 / 2-h$ testing sessions. On Days 2, 4, and 6, the animals were deprived of food for $23 \mathrm{~h}$ prior to testing. 


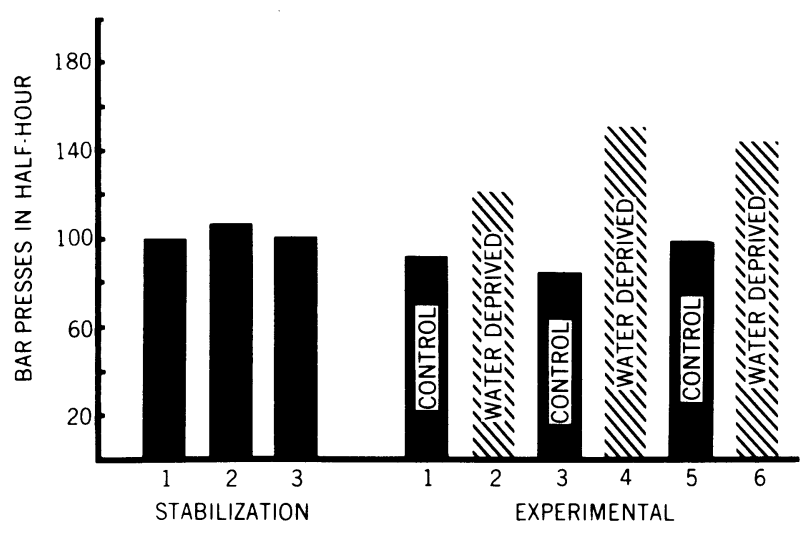

Figure 3. Mean barpresses for hippocampal stimulation in $1 / 2-h$ testing sessions. On Days 2,4 and 6, the animals were deprived of water for $23 \mathrm{~h}$ prior to testing.

rates when water-deprived than under ad-lib water. This increase occurred at the current selected initially for seven animals. In one case, the current had to be lowered three times before a deprivation effect occurred. The one animal which did not show an increase in barpressing was tested at only two current levels, as a lower current failed to support stable barpressing. The rate increase observed in the group of nine animals was significant (correlated means, $\mathrm{t}=5.15$, df $=8, \mathrm{p}<.01$, two-tailed). Figure 3 summarizes the data for all nine animals.

This experiment shows that hippocampal selfstimulation can be facilitated by either food or water deprivation. Although the increase under food deprivation may be related to the facilitatory effect of hippocampal stimulation on feeding, the increase under water deprivation is more difficult to understand, since electrical stimulation of the dorsolateral hippocampus does not facilitate drinking (Milgram, $1969 \mathrm{~b})$. On the other hand, hippocampal involvement in the control of drinking is suggested from studies using other techniques (e.g., Grant \& Jarrard, 1968), and it is conceivable that electrical stimulation could facilitate a reward system associated with intake of water, but not elicit drinking.

\section{EXPERIMENT 2}

Since Experiment 1 established that food and water deprivation each cause an increase in hippocampal self-stimulation, the purpose of Experiment 2 was to determine the effects of these manipulations on poststimulation hippocampal feeding.

\section{Experiment 2a Effect of Food Deprivation on Poststimulation Hippocampal Feeding \\ Methods \\ The subjects were five male Long-Evans rats implanted with dorsolateral hippocampal electrodes, using the stereotaxic coordinates described in the first experiment.}

The animals were tested in the Skinner boxes previously described, except that the bar was disconnected from the stimulator. The initial phase involved six $1 / 2-\mathrm{h}$ adaptation sessions to the testing boxes under 6-h food deprivation. A weighed quantity of Purina laboratory pellets was placed in a metal cup at one end of the box, and water was provided in a graduated cylinder at the opposite end.

Testing involved $1 / 2-h$ measurements of food intake under four conditions, in the following order: Day 1 (stimulation-deprivation), the animals received programmed hippocampal stimulation and were food deprived for $6 \mathrm{~h}$; Day 2 (deprivation alone), the animals were deprived of food, but the stimulator was set to zero; Day 3 (stimulation alone), the animals received stimulation, and were not deprived of food; Day 4 (control), the animals did not receive stimulation and were not food deprived. This 4-day sequence of testing was repeated five times for each rat. During the stimulation sessions, the rats received a $1-\mathrm{sec}$ train of $0.1-\mathrm{msec}$ duration $100-\mathrm{Hz}$-frequency square-wave pulses at an intensity of $250 \mu \mathrm{A}$ delivered once every $30 \mathrm{sec}$; thus, the stimulation condition consisted of 601 -sec trains of square-wave pulses.

\section{Results and Discussion}

Figure 4 illustrates that food deprivation interacts with hippocampal stimulation; the animals ate significantly more under the stimulation-deprivation condition than they did under the deprivation-alone condition (correlated means, $\mathrm{t}=5.2, \mathrm{df}=4, \mathrm{p}<.01$, two-tailed) or under the stimulation-alone condition (correlated means, $\mathrm{t}=7.79$, df $=4, \mathrm{p}<.01$, two-tailed). Intake during the stimulation-alone condition was significantly greater than during the control condition (correlated means, $t=2.88$, $\mathrm{df}=4, \mathrm{p}<.05$, two-tailed).

As is apparent from Figure 4, the food intake during the combined stimulation-deprivation condition was considerably greater than that during any of the other conditions, and a comparison of feeding under the stimulation-deprivation condition with the sum of the feeding under the stimulation condition and deprivation condition proved to be statistically

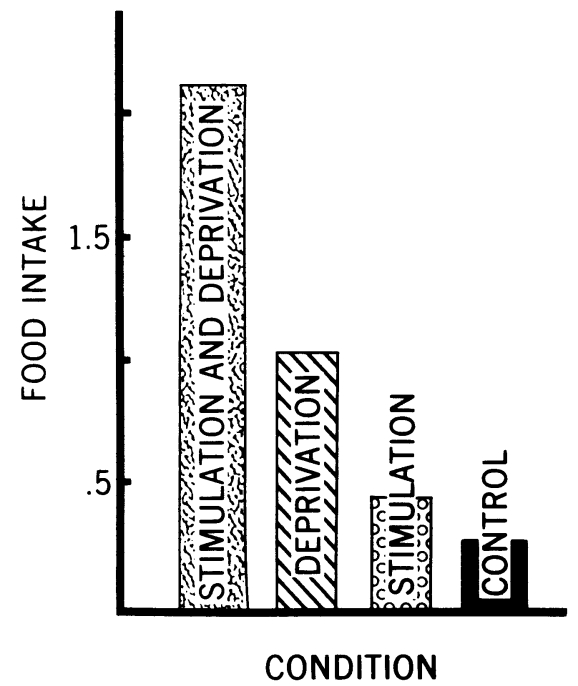

Figure 4. Mean food intake in $1 / 2-h$ sessions under $6 \mathrm{~h}$ of food deprivation and/or programmed hippocampal stimulation. The control condition indicates feeding when nondeprived and without stimulation. 
significant (correlated means $t=5.34, \mathrm{df}=4$, $\mathrm{p}<.01$, two-tailed).

\section{Experiment 2b Effect of Water Deprivation Methods on Poststimulation Hippocampal Feeding}

The subjects were nine naive male Long-Evans rats with single dorsolateral hippocampal electrodes. Food, but not water, was available in the testing box. The animals received intermittent hippocampal stimulation delivered from a $60-\mathrm{Hz}$ sine-wave stimulator set at $30 \mu \mathrm{A}$ and programmed to deliver a 1-sec stimulus every $30 \mathrm{sec}$ during the stimulation tests.

The experimental testing began on Day 7 and continued until Day 18, as in the schedule of Experiment 2a, but with a 6-h waterdeprivation period instead of food deprivation.

\section{Results and Discussion}

Six hours of water deprivation had little effect on poststimulation feeding (Figure 5). Food intake during the stimulation-alone condition was slightly greater than that during the combined stimulationdeprivation condition, but the difference was not statistically significant.

The results of this experiment indicate that deprivation influences hippocampal feeding in a manner consistent with the influence of deprivation on normal intake. Experiment $2 \mathrm{a}$ demonstrated that mild food deprivation interacts with hippocampal stimulation to produce substantially more feeding than either of the two conditions separately. Water deprivation, in contrast, produced a slight decrease in poststimulation feeding which was not surprising in view of evidence that water deprivation suppresses normal feeding (e.g., Verplanck \& Hayes, 1953).

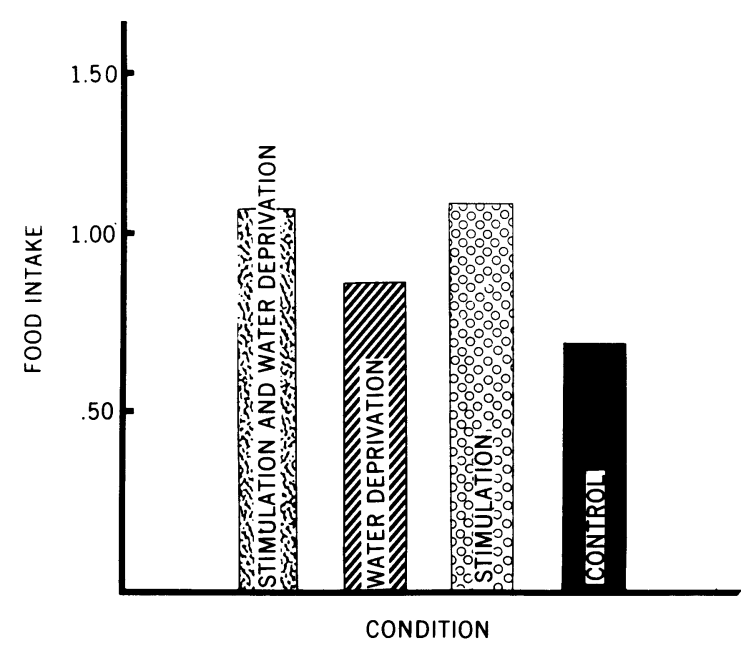

Figure 5. Mean food intake in $1 / 2-\mathrm{h}$ sessions under $6 \mathrm{~h}$ of water deprivation and/or programmed hippocampal stimulation. Neither deprivation nor stimulation were present during the control condition.

\section{EXPERIMENT 3 EFFECT OF DEPRIVATION ON HYPOTHALAMIC SELF-STIMULATION}

One possible explanation for the facilitation of hippocampal self-stimulation by food or water deprivation is that the increased barpressing is a nonspecific consequence of the arousal resulting from deprivation. This suggestion is unlikely in view of other research indicating that water deprivation has no effect on hypothalamic self-stimulation (e.g., Annau \& Baetjer, 1971; Carey, Goodall, \& Lorens, 1975; Mogenson, 1969). It is, nevertheless, possible that a deprivation effect might have been obscured by the procedures used in these studies. We therefore used a testing paradigm similar to that used in Experiment 1 in order to examine the effect of food or water deprivation on hypothalamic self-stimulation.

\section{Methods}

The subjects were 10 male Long-Evans rats with monopolar electrodes implanted in the lateral hypothalamic area, using stereotaxic coordinates $1.75 \mathrm{~mm}$ lateral to the midline, $6.0 \mathrm{~mm}$ anterior to the ear bars, and $7.4 \mathrm{~mm}$ down from the dura.

The testing apparatus was similar to that described for the first experiment, except that a $60-\mathrm{Hz}$ sine-wave stimulator was used to electrically stimulate the brain. There were five phases to the experiment: training, stabilization, food deprivation, water deprivation, and tests for elicited behaviors.

A week following surgery, the animals were shaped to barpress for $0.5 \mathrm{sec}$ duration hypothalamic stimulation. During the remainder of the training phase, the animals were given daily $1 / 2-h$ barpressing sessions, during which the stimulation intensity was adjusted to a level which supported barpressing rates between 20 and 30 presses/min (range $5-45 \mu \mathrm{A}$ rms).

The stabilization phase began after selection of an appropriate current intensity. The daily barpressing sessions continued at the selected current intensity until the deviation of any session from the mean of the three sessions was less than $10 \%$ over three consecutive sessions. Half of the animals were subsequently tested for the effect of food deprivation on self-stimulation, and half of the animals were tested for the effect of water deprivation on hypothalamic self-stimulation. The method used was as described in Experiment 1. Under each condition, the current intensity was lowered up to three times in those cases where no deprivation effect was observed. For the purpose of statistical analysis, the data were based either on barpressing at the current level for which an effect was observed or on barpressing at the lowest current tested.

At the end of this part of the experiment, each animal was retested, but under the other deprivation condition. Thus, those animals initially tested under food deprivation were then tested under water deprivation, and vice versa.

At the conclusion of self-stimulation testing, the animals were tested for the effects of hypothalamic stimulation on feeding and drinking over five $1 / 2-h$ sessions. A self-induced feeding and drinking procedure was used as described by Devor, Wise, Milgram, and Hoebel (1970). The animals were trained to barpress for long durations $(20 \mathrm{sec})$ of continuous hypothalamic stimulation and then tested for 5 consecutive days with food and water both available in the Skinner box.

\section{Results}

Effects of food and water deprivation on hypo- 
thalamic self-stimulation. The data were analyzed in a split-plot factorial design analysis of variance which revealed a significant interaction between deprivation and food vs water. The origin of this interaction is shown in Table 1. Comparisons between pairs of means revealed a significant increase in barpressing under food deprivation compared to the corresponding control days $(t=4.73$, $\mathrm{df}=8, \mathrm{p}<.01$, two-tailed). In contrast, barpressing under water deprivation was not significantly different from the control sessions $(t=1.82$, $\mathrm{df}=8, \quad \mathrm{p}>.10$, two-tailed). Even a cursory examination of the individual data illustrates this effect: 9 of 10 animals showed an increase under food deprivation; under water deprivation, five animals pressed at a slightly higher rate and five animals pressed at a lower rate.

Relationship to elicited behaviors. All of the animals showed stimulation-bound feeding and 8 of the 10 rats were also stimulus-bound drinkers. There were marked individual differences; in some cases, the elicited drinking was considerably more vigorous, in other instances just the opposite was true.

In order to determine whether there was any relationship between the elicited behaviors and the effects of deprivation on barpressing, Pearson correlations were calculated between the quantity consumed during each elicited behavior and the percentage change in barpressing under each kind of deprivation. Table 2 shows that these relationships were all small and not significant.

\section{GENERAL DISCUSSION}

The present series of experiments indicates that the reinforcing consequences of hippocampal stimulation are at least partially independent of the effects of hippocampal stimulation on feeding. Although hippocampal self-stimulation and poststimulation feeding were similarly increased under food deprivation, water deprivation caused a facilitation of selfstimulation but had no effect on feeding. The selective effect of water deprivation on barpressing could not be attributable to energizing or other nonspecific consequences of deprivation on selfstimulation. Barpressing for lateral hypothalamic stimulation was not affected by water deprivation, indicating a specificity of the water-deprivation effect to the hippocampus. Another possibility is that the increase in barpressing was a consequence of the effect of water deprivation on feeding. Since water deprivation is known to inhibit feeding (Verplanck \& Hayes, 1953), food and water deprivation may have increased barpressing for the same reason. This explanation is unlikely in view of the failure of water deprivation to affect hypothalamic self-stimulation; barpressing for hypothalamic stimulation was facilitated by food deprivation.
Table 1

Mean Barpresses Over $1 / 2-h$ Sessions for Hypothalamic Animals Under Food Deprivation, Water Deprivation, or Control (Ad-Lib) Conditions

\begin{tabular}{ccc}
\hline & \multicolumn{2}{c}{ Testing Phase } \\
\cline { 2 - 3 } Testing & $\begin{array}{c}\text { Food } \\
\text { Deprivation }\end{array}$ & $\begin{array}{c}\text { Water } \\
\text { Deprivation }\end{array}$ \\
\hline Ad-Lib & 1503 & 1537 \\
Deprived & 1806 & 1420 \\
\hline
\end{tabular}

Table 2

Correlation Coefficients Between Elicited Hypothalamic Behaviors and Change in Barpressing Under Deprivation

\begin{tabular}{ccc}
\hline & \multicolumn{2}{c}{$\%$ Change in Barpressing } \\
\cline { 2 - 3 } $\begin{array}{c}\text { Elicited } \\
\text { Behavior }\end{array}$ & $\begin{array}{c}\text { Food } \\
\text { Deprivation }\end{array}$ & $\begin{array}{c}\text { Water } \\
\text { Deprivation }\end{array}$ \\
\hline Feeding & -.14 & +.17 \\
Drinking & +.04 & -.19 \\
\hline
\end{tabular}

The finding that hypothalamic stimulation was facilitated by food but not water deprivation is consistent with other research (e.g., Carey et al., 1975), and indicates that the dissociation between selfstimulation and electrically elicited behaviors is not limited to the hippocampus. As was found in Experiment 3, lateral hypothalamic stimulation can generally be shown to elicit both feeding and drinking (Milgram et al., 1971), and each elicited behavior is appropriately modified by manipulations which affect the corresponding behavior of normal animals (e.g., Devor et al., 1970). Thus, water deprivation facilitates drinking elicited by lateral hypothalamic stimulation, but has no effect on self-stimulation through the same electrode.

The present findings are also relevant to the question of the relationship between the reinforcing consequences of brain stimulation at different neural loci. Several investigators have proposed either a common neuroanatomical or neurochemical basis for self-stimulation (e.g., German \& Bowden, 1974; Olds \& Olds, 1965). Consistent with such speculation, Milgram (1968) suggested that activation of hypothalamic mechanisms may account for the reinforcing consequences of hippocampal stimulation. This line of speculation leads to the prediction that events which modify hippocampal reward should have a corresponding effect on hypothalamic reward, a prediction which was not confirmed because of the selective action of water deprivation. Campbell (1975) reported further evidęnce indicating a dissociation between hippocampal and hypothalamic reward: hippocampal self-stimulation persisted after virtually complete section of the fornix, the hippocampal efferent pathway to the hypothalamus. More generally, such data suggest that the diversity in the reinforcing consequences of brain stimulation may be greater than has generally been assumed. Other 
recent evidence lends further credence to this view: the effects of both amphetamine and food deprivation on self-stimulation vary markedly between different electrode loci (Carey et al., 1975; Goodall \& Carey, 1975).

\section{REFERENCES}

Annau, Z., \& BaEtJer, A. M. Effects of prolonged water deprivation on hypothalamic self-stimulation. American Journal of Physiology, 1971, 220, 83-86.

BALL, G. G. Separation of electrical self-stimulation and electrically elicited eating in the hypothalamus. Communications in Behavioural Biology, 1969, 3, 5-10.

CAMPBELL, K. A. Motivational consequences of hippocampal and septal stimulation: Role of the fornix. Unpublished MA thesis, University of Toronto, 1975.

Carey, R. J., Goodall, E., \& Lorens, S. A. Differential effects of amphetamine and food deprivation on self-stimulation of the lateral hypothalamus and medial frontal cortex. Journal of Comparative and Physiological Psychology, 1975, 88, 224-230.

Devor, M., Wise, R., Milgram, N. W., \& Hoebel, B. G. Physiological control of hypothalamically elicited feeding and drinking. Journal of Comparative and Physiological Psychology, 1970, 73, 226-232.

Deutsch, J. A., \& Deutsch, D. Physiological psychology. Homewood, Ill: Dorsey Press, 1973.

German, D. C., \& Bowden, D. M. Catecholamine systems as the neural substrate for intracranial self-stimulation: A hypothesis. Brain Research, 1974, 73, 381-419.

Goldstein, R., Hill, S., \& Templer, D. I. Effects of food deprivation on hypothalamic self-stimulation in stimulus- bound eaters and non-eaters. Physiology and Behavior, 1970, 5, 915-918.

Goodall, E. B., \& Carey, R. J. Effects of d- versus l-amphetamine, food deprivation, and current intensity on self-stimulation of the lateral hypothalamus, substantia nigra, and medial frontal cortex of the rat. Journal of Comparative and Physiological Psychology, 1975, 89, 1029-1045.

Grant, L. P., \& JARRARD, L. E. Functional dissociation within hippocampus. Brain Research, 1968, 10, 392-401.
Hoebel, B. G. Feeding: Neural control of intake. Annual Review of Physiology, 1971, 33, 533-568.

Margules, D. L., \& Olds, J. Identical "feeding" and "rewarding" systems in the lateral hypothalamus of rats. Science, 1962, 135, 374-375.

Milgram, N. W. Effect of hippocampal stimulation on feeding in the rat. Unpublished doctoral dissertation, McGill University, 1968.

Milgram, N. W. Effect of hippocampal stimulation on feeding in the rat. Physiology and Behavior, 1969, 4, 665-670. (a)

Milgram, N. W. Motivational specificity of dorso-lateral hippocampal stimulation. Communications in Behavioral Biology, 1969, 4, 1-5. (b)

Milgram, N. W.,-Devor, M., \& Server, A. C. Spontaneous changes in behaviors induced by electrical stimulation of the lateral hypothalamus in rats. Journal of Comparative and Physiological Psychology, 1971, 75, 491-499.

Mogenson, G. J. Water deprivation and excessive water intake during self-stimulation. Physiology and Behavior, 1969, 4, 393-397.

Mogenson, G. J., \& Stevenson, J. A. F. Drinking induced by electrical stimulation of the lateral hypothalamus. Experimental Neurology, 1967, 17, 119-127.

Olds, J., \& Olds, M. E. Drives, rewards, and the brain. In T. M. Newcombe (Ed.), New directions in psychology (II). New York: Holt, Rinehart, \& Winston, 1965.

Oliver, D. L., Firestone, M. I., \& Goodman, E. D. Hippocampal stimulation rebound effects in hamsters. Physiology and Behavior, 1973, 10, 23-27.

Trowill, J. A., Panksepp, J., \& Gandelman, R. An incentive model of rewarding brain stimulation. Psychological Review, 1969, 76, 264-281.

Valenstein, E. S., Cox, V. C., \& Kakolewski, J. W. Re-examination of the role of the hypothalamus in motivation. Psychological Review, 1970, 77, 16-31.

Verplanck, W. S., \& HaYes, J. R. Eating and drinking as a function of maintenance schedule. Journal of Comparative and Physiological Psychology, 1953, 46, 773-776.

(Received for publication August 31, 1976; revision accepted October $28,1976$. 